\title{
The asteroid belt outer region under jumping-Jupiter migration
}

\author{
H. S. Gaspar, ${ }^{1,2 \star}$ O. C. Winter ${ }^{2 \star}$ and E. Vieira Neto ${ }^{2 \star}$ \\ ${ }^{1}$ Univ. Federal de Santa Catarina, R. Dr. João Colin, 2700, Joinville, SC, CEP 89 218-035, Brazil \\ ${ }^{2}$ Univ. Estadual Paulista. Grupo de Dinâmica Orbital e Planetologia, Av. Dr. Ariberto Pereira da Cunha, 333, Guaratinguetá, SP, CEP 12 516-410, Brazil
}

Accepted 2017 May 16. Received 2017 May 12; in original form 2016 February 11

\begin{abstract}
The radial configuration of the outer region of the main asteroid belt is quite peculiar, and has much to say about the past evolution of Jupiter. In this work, we investigate the dynamical effects of a jumping-Jupiter-like migration over a more extended primordial asteroid belt. Jupiter's migrations are simulated using a fast jumping-Jupiter synthesizer. Among the results, we highlight non-negligible fractions of primordial objects trapped in 3:2 and 4:3 mean motion resonances (MMRs) with Jupiter. They survived the whole truculent phase of migration and originated populations that are like Hildas and Thules. Fractions ranging from 3 to 6 per cent of the initial distribution remained trapped in 3:2 MMR, and at least 0.05 percent in 4:3. These results show that the resonance trapping of primordial objects may have originated these resonant populations. This theory is consistent even for Jupiter's truculent evolution.
\end{abstract}

Key words: minor planets, asteroids: general-planet-disc interactions.

\section{INTRODUCTION}

There is a consensus that the Solar system's giant planets were formed in different orbits than the ones observed today. The orbits currently observed resulted from planetary migrations. Planets migrate due to angular momentum exchange with either the gaseous circumsolar disc (gas-driven) or other objects, such as a disc of planetesimals for instance (planetesimal-driven). Further details on gas-driven migration are given by Ward (1997). The planetesimaldriven migration scenario was first proposed by Fernandez \& Ip (1984) and further explored by others (e.g. Hahn \& Malhotra 1999; Gomes, Morbidelli \& Levison 2004). Comprehending how such an evolution occurred is still a challenge. However, the Solar system (hereafter SS) was sculpted by each one of its components. In other words, comprehending which processes originated the currently observed features of the SS means looking through a window into the past.

The SS's two main reservoirs of small bodies provide important constraints on the planetary evolution. The main asteroid belt is located in the inner part of the SS while the Kuiper belt is in the outer region. Whilst many features of the outer SS configuration are explained well by the original Nice model (Tsiganis et al. 2005; Morbidelli et al. 2005), much of the current efforts are devoted to comprehending the processes that must have sculpted the inner SS (Minton \& Malhotra 2009; Brasser et al. 2009; Walsh et al. 2011). The main asteroid belt has a structure as complex as that of the Kuiper belt. Hence, considerable attention has been devoted to it. The outermost region of the main asteroid belt stands as a witness of

^E-mail: helton.s.gaspar@ufsc.br (HSG); ocwinter@feg.unesp.br (OCW); ernesto@feg.unesp.br (EVN) the past evolution of Jupiter. Let us precisely define the outermost region as $a>a_{2: 1}$, i.e. the region beyond the gap associated with the $2: 1$ mean motion resonance (MMR) with Jupiter, where $a_{2: 1} \approx$ 3.28 au. Fig. 1 evinces the role of Jupiter over the complex structure of this outermost region:

(i) A gap associated with the 2:1 MMR with Jupiter.

(ii) A cluster located beyond the locus of the 2:1 MMR.

(iii) A depleted region ranging from 3.6 to $3.9 \mathrm{au}$. Seven of the few objects within this depleted region seem to have had their eccentricities excited by the 5:3 and the 8:5 MMRs.

(iv) The Hilda group of asteroids clustered around the locus of 3:2 MMR. Hildas comprise roughly 20 per cent of the objects in the figure.

(v) Another depleted region beyond that populated by the Hildas, $4.05<a<4.25$ au.

(vi) A small resonant population at the locus of 4:3 MMR. The three components of the dynamical group Thule are labelled.

This paper presents a study of the consequences for the outer region of a primordial asteroid belt due to Jupiter's migration. In the context of the classic smooth planetesimal-driven migration conjuncture (e.g. Fernandez \& Ip 1984), this issue was studied by Franklin et al. (2004). In particular, they show that the peculiar characteristics of Hildas can result from captured field asteroids if Jupiter migrated sunward by about 0.4 au over a time greater than $10^{5} \mathrm{yr}$.

Such a classic migration scenario is currently in check. One of the reasons is that a slow and smooth migration of Jupiter implies a slow sweeping of secular resonances across the inner SS, resulting in a slight instability (Minton \& Malhotra 2009; Brasser et al. 2009). Hence, the jumping-Jupiter migration scenario (Brasser et al. 2009; 


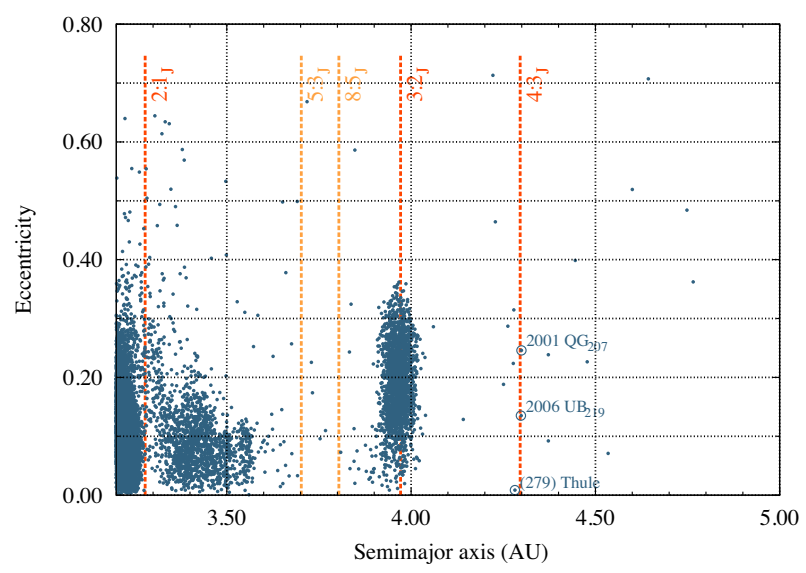

Figure 1. Outermost region of the main asteroid belt $\left(a>a_{2: 1}\right)$. Distribution of objects with more than three oppositions. Vertical dashed lines highlight the loci of five MMRs with Jupiter (source: http://www.minorplanetcenter.net; Holman et al. 2015).

Morbidelli et al. 2010), addressed in Section 2, is a broadly accepted alternative.

The jumping-Jupiter scenario is considered in this work. We aim to assess whether a more extended primordial outer belt can result in a radial structure like the one currently observed for the outer region of the main asteroid belt. In particular, we intend to verify the following:

(i) Can the primordial belt objects be trapped in resonances?

(ii) Can the objects trapped in resonance migrate together with their respective resonance loci?

(iii) Will the region occupied by the primordial belt be depleted, resulting in the gaps mentioned?

In Section 2, we discuss the assumptions for the adopted migration scenario. Details about the mimicked migration algorithm can be found in Appendix A. The results are split into three subsections. In Section 3.1, we discuss how the initial scenario evolves in time and Section 3.2 addresses the final configuration. Section 3.1.1 presents the analysis on the effects of an orbital leap by Jupiter. The conclusion and final considerations are addressed in Section 4.

\section{THE MIGRATION SCENARIO}

In Morbidelli et al. (2009) and Brasser et al. (2009), the authors describe a migration mechanism where one ice giant planet, Neptune or Uranus, suffers two fateful planetary encounters. The first encounter with Saturn causes the icy planet to be thrown sunward, as the result of an orbital lift of the gaseous planet. The second encounter, with Jupiter, results in its outward scattering causing Jupiter's orbit to shrink. Hence, these two encounters cause a rapid increase in the mutual separation of the gaseous giant planets, since, as a reaction, Saturn's orbit is expanded and the Jupiter's orbit is shrinked. This rapid increase in the separation of the giant planets avoids the mentioned inner SS instability, which arises when Jupiter and Saturn take a substantial time to cross their mutual 2:1 MMR. This mechanism is known as jumping-Jupiter. Nesvorný (2011) also proposed an alternative way of achieving it. In this similar proposal, a supposed fifth Neptune-sized giant planet is ejected from the SS during the second encounter with Jupiter.

Aiming to answer those questions in Section 1, a spline interpolation algorithm was adopted to mimic the time evolution of the
Jovian semi-major axis. We did not interpolate a real simulation of jumping Jupiter. Instead, we developed an algorithm to synthesize a stochastic semi-major axis evolution like that shown in fig. 5 of Morbidelli et al. (2010). The details of the spline algorithm are summarized in Appendix A. Morbidelli et al. (2010) states that the Jupiter-Saturn mutual separation must occur on a time-scale of 10$10^{2} \mathrm{kyr}$. Here, we perform tests for three time-scales: 10, 50 and $100 \mathrm{kyr}$. For each of these, we perform three different evolutions of the semi-major axis, each corresponding to three distinct seed values, hence resulting in nine combinations, since distinct seed values result in distinct evolutions. To simplify, Jupiter's eccentricity is kept constant at either 0.0 or 0.06 . One can see in fig. 5 of Morbidelli et al. (2010) that the Jovian osculating eccentricity barely surpasses the upper limit of 0.06 . The migration scenario in which Jupiter's eccentricity is kept constantly as high as 0.06 must result in additional instability of the main asteroid belt than a scenario in which Jupiter's eccentricity varies in time within the range [0:0.06].

Saturn is not taken into account in our experiments. As pointed out by Franklin et al. (2004), the main effect of Saturn is the excitation of the Jovian eccentricity, but there is no substantial direct effect on the outer region of the main belt. Nevertheless, in this work the Jovian orbital eccentricity is kept constant. We performed some experiments with Saturn to infer that it does not affect the populations studied. Saturn's orbit was set to 8.5 au and its eccentricity to 0.1 . These are the lower semi-major axis and higher eccentricity of Saturn in fig. 5 of Morbidelli et al. (2010).

The supposed fifth icy giant is not considered in our work. The history of the fifth giant is supposed to have been highly chaotic. A planet in such circumstances must not affect any particular asteroidal region of the asteroid belt because it does not remain on any nominal orbit during a substantial time. Instead, it is just expected to scatter those asteroids that cross its orbit. The work by Roig \& Nesvorný (2015) takes into account the effects of a fifth icy giant on the main asteroid belt. From their fig. 4, one infers that Jupiter is at $\sim 5.07$ au so that the $3: 2 \mathrm{MMR}$ locus is at $\sim 3.87 \mathrm{au}$, where there exist some remaining objects. Therefore, their results confirm that the effects due to the fifth icy giant do not decimate such a resonant population.

The whole set of experiments consists of 18 simulations. To facilitate the reference to each simulation, we employed a ternary tag TSE, where T (10, 50 or 100) matches the time-scale, S (A, B or $C$ ) stands for the three distinct seed parameters and $E$ ( 0 or 6 ) matches the Jovian eccentricities. Fig. A2 in the appendix contains the nine distinct evolutions labelled with their respective TSE tag. For these experiments, we uniformly distribute 3000 test particles within the $a \times e \times I$ space defined by:

$$
\left\{\begin{aligned}
a_{3: 2}-0.60 \mathrm{au} & \leqslant a \leqslant 0.60 \mathrm{au}+a_{3: 2} \\
0.0 & \leqslant e \leqslant 0.30 \\
0.0 & \leqslant I \leqslant 1.00^{\circ},
\end{aligned}\right.
$$

where $a, e$ and $I$ stand for the osculating semi-major axis, eccentricity and inclination of the test particles, respectively, and, hereafter, $a_{3: 2}$ stands for the dynamical locus of the 3:2 MMR. Therefore, for Jupiter with orbital semi-major axis $a_{\mathrm{J}}=5.4 \mathrm{au}$, one has $a_{3: 2} \approx$ $4.12 \mathrm{au}$. The initial distributions of the test particles are identical for each of the 18 experiments. In this work, we are interested in evaluating the results within the radial distribution scope. Hence, we limited the maximum initial inclination to $1^{\circ}$, which is enough. We also performed a simulation with 6000 test particles with inclinations uniformly distributed up to $20^{\circ}$. The results are like those presented in this paper, but a larger number of test particles would be necessary to improve the sampling. Simulations for these systems 


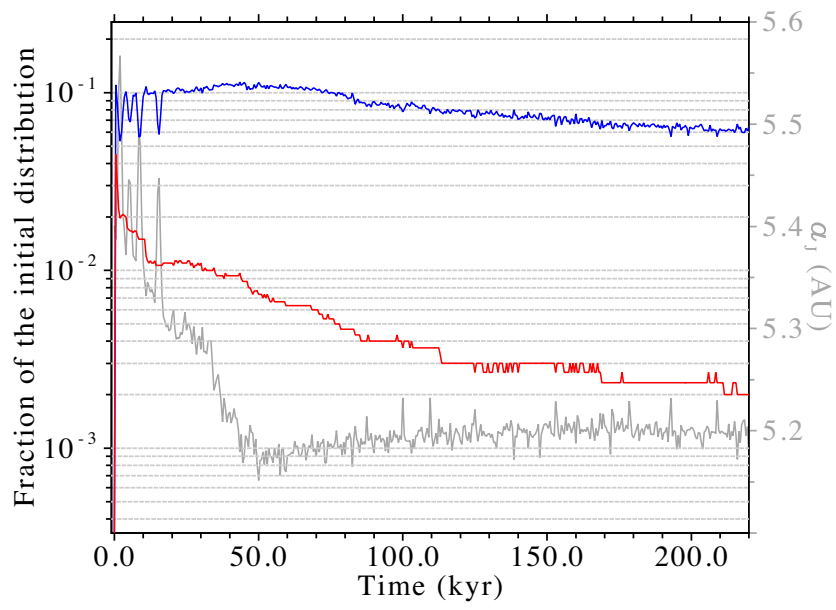

Figure 2. Fractions of asteroids in 3:2 in blue and 4:3 MMR in red. For comparison, Jupiter's semi-major axis is depicted in grey. Data from experiment $50 \mathrm{~A} 0$.

are carried out for $2.5 \times 10^{2} \mathrm{kyr}$ using a Gauss-Radau time step integrator (Everhart 1985), with an upper limit of the time step of $10^{-2}$ yr. We used a c (Ritchie \& Kernighan 1978) implementation of the FORTRAN (IBM 1954) version published by Everhart (1985). This integrator has the advantage of been a reliable and quick secondorder integrator. To save computing time, test particles are discarded once they escape from the following region of interest:

$a_{3: 2}-1.0 \mathrm{au} \leqslant a \leqslant a_{\mathrm{J}}$.

Note that such a region is dynamically defined since both the semimajor axis of Jupiter $a_{\mathrm{J}}$ and the locus of the 3:2 MMR $a_{3: 2}$ vary in time. The results of these experiments are presented in Section 3.1.

\section{RESULTS}

\subsection{Consequences of a jumping-Jupiter-like migration}

This section presents the results of experiments performed to assess whether:

(i) Asteroids in the outer region of the primordial belt can become trapped in resonance.

(ii) A substantial fraction remains trapped throughout the Jupiter migration.

In this context, objects trapped in resonance are those whose orbital semi-major axis $a$ is comparable to the resonance orbital locus $a_{m: n}$ and the resonant angle $\phi$ is librating. More precisely, $\left|a-a_{m: n}\right|<0.1$ au must be satisfied. By using the definition in Murray \& Dermott (1999), one can derive the following resonant angle:

$\phi=m\left(M^{\prime}+\varpi^{\prime}-\varpi\right)-n M$,

where $m$ and $n$ are the resonance commensurable values, and $M$ and $\varpi$ are the asteroid's mean anomaly and longitude of perihelion, respectively. The primed quantities $M^{\prime}$ and $\varpi^{\prime}$ are the analogous values for Jupiter.

Fig. 2 shows the fractions of resonant asteroids throughout the integration time superimposed over the semi-major axis of Jupiter for comparison. The observed noise means that some asteroids alternate between the libration and circulation regimes. Such regime transitions are due to the noisy migration of Jupiter. The rapid variations

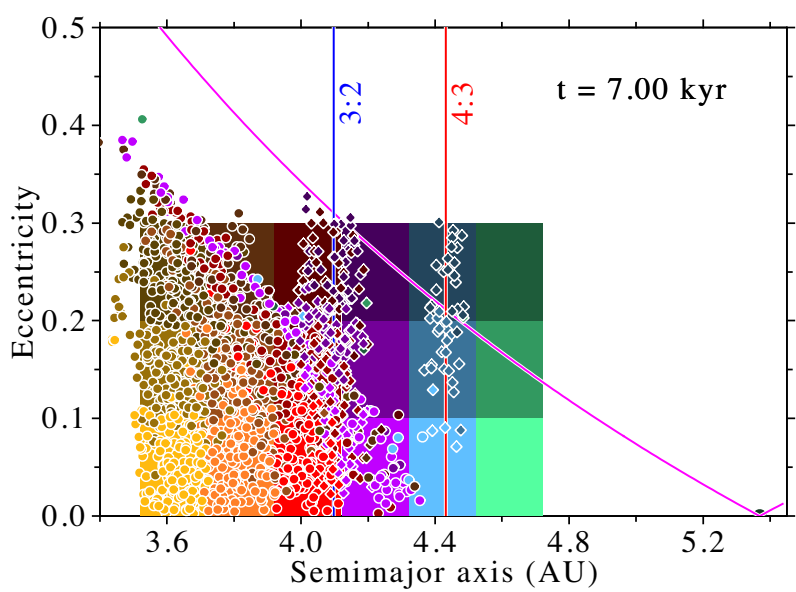

Figure 3. Snapshot of the $a \times e$ distribution for the experiment $50 \mathrm{~A} 0$ at $t=7 \mathrm{kyr}$. Coloured bullets stand for test particles. The colour matches the source region in the plot. Diamonds stand for objects trapped in resonance. Jupiter is depicted by the black bullet. The resonance loci are marked with vertical lines. The perihelion and aphelion curves are in magenta.

of the orbital semi-major axis of Jupiter imply a fast variation of the orbital period, and a consequent breaking of the commensurability with some objects. This is quite evident in the first $15 \mathrm{kyr}$, during which the evolution of Jupiter's semi-major axis presents four leaps. For each of these leaps, note the remarkable losses of objects in 3:2 MMR (blue curve) concomitant with the orbital raising of Jupiter. However, during the following orbital shrinkage, most of the lost objects become trapped back in resonance. Section 3.1.1 presents the results of experiments for orbital leaps in further detail.

Fig. 2 can also be analysed in relation to the average profile of its curves. Notice that the fraction of objects in 3:2 MMR increases to more than 10 per cent at $t \sim 45 \mathrm{kyr}$. This is a consequence of the main phase of migration. As Jupiter migrates from an initial average orbit of 5.4 au to its final average orbit of $5.2 \mathrm{au}$, the resonance locus moves from 4.12 to $3.97 \mathrm{au}$. Consequently, some asteroids from the field swept by the resonance locus become resonant during this main migration phase.

The profile of the red curve in Fig. 2 differs substantially from that discussed for the blue curve. Essentially, this is because the locus of the 4:3 MMR resonance sweeps a field devoid of asteroids. As the average orbit of Jupiter shrinks from 5.4 to 5.2 au, the $a_{4: 3}$ sweeps the field from 4.46 down to $4.29 \mathrm{au}$. Therefore, a negligible fraction of new objects is captured in 4:3 resonance, whilst leakage continues due to the noisy migration. Fig. 3 shows a snapshot of the system distribution in $a \times e$ space at $t=7 \mathrm{kyr}$. At this instant, Jupiter's orbit is near to $5.4 \mathrm{au}$. This plot depicts the field devoid of asteroids between $a_{3: 2}$ and $a_{4: 3}$, which is the sparse field that will be swept by the locus of the 4:3 MMR.

The history of the test particles trapped in resonance is investigated to distinguish those that never escape the resonance. For the case shown in Fig. 2, 28.3 per cent of the accomplished captures in 3:2 MMR were effective, i.e. a fraction of about 2 per cent of the total population was once trapped in 3:2 MMR and continued to librate until the end of the simulation. We observed that the penultimate effective trapping occurred after only $200 \mathrm{yr}$ of the beginning of the simulation, and the last one occurred $34.14 \mathrm{kyr}$ after the penultimate one. By effective trapping, we mean that an object never left the librating regime since its first resonance trapping. For the case 4:3 MMR in Fig. 2. The total 0.2 percent of trappings 


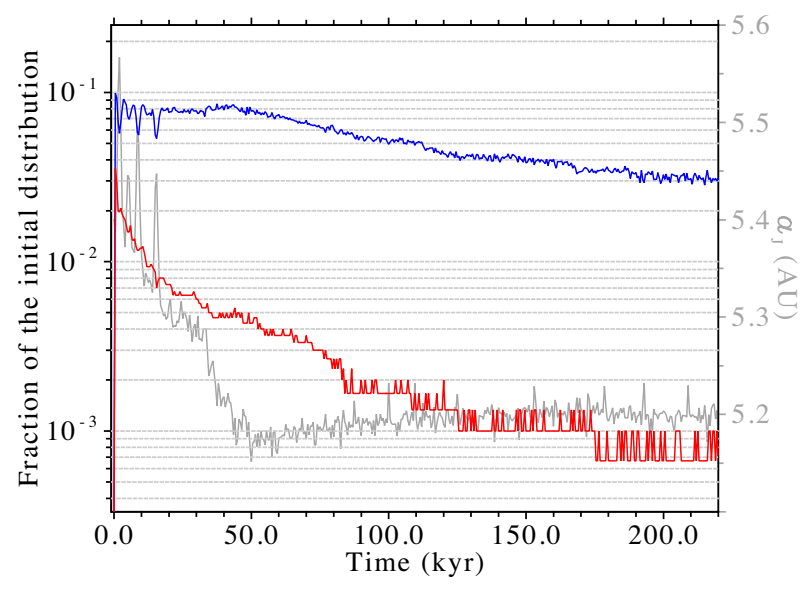

Figure 4. Analogous to Fig. 2, but for Jupiter in an eccentric orbit with $e=0.06$.

shown in the plot were effective trappings. The last trapping in 4:3 MMR occurred only $160 \mathrm{yr}$ after the beginning of the simulation.

Fig. 4 contains the results of an experiment analogous to that of Fig. 2 but for Jupiter in an eccentric orbit, i.e. all the parameters of the simulation are the same except that Jupiter's eccentricity is held equal to 0.06 throughout the whole simulation. The results shown in Fig. 4 are like those in Fig. 2, in the following sense:

(i) Jupiter's noisy migration also causes the asteroids to leave the libration regime.

(ii) Resonance 3:2 accomplishes new captures as its locus sweeps over the internal asteroid field.

(iii) Resonance 4:3 does not accomplish significant new captures during the migration because its locus sweeps a field devoid of asteroids.

On the other hand, notice the remarkable difference in the numbers of objects trapped in both the 3:2 and 4:3 MMR resonances. Jupiter's eccentricity results in a reduction of the fraction of resonance trappings. This is because the most elongated orbit of Jupiter has a larger number of test particles crossing it. Hence, a larger number of test particles are removed by encounters with Jupiter. Fig. 5 illustrates this discussion. Essentially, all those test particles above the Jovian aphelion curve are supposed to be removed.

For the simulation shown in Fig. 4, 18 per cent of the resonance trappings in 3:2 MMR were effective (they never stopped librating). This number corresponds to about 0.54 per cent of the total population.

The last effective capture in 3:2 MMR occurred $720 \mathrm{yr}$ after the beginning of the simulation. All those 0.066 per cent of the total population trapped in 4:3 MMR were effective by the time $t=160 \mathrm{yr}$, at the latest.

Analogous results were also observed in the other experiments. To summarize all the results, we compiled the main data in the plot of Fig. 6. The panels summarize the percentages of resonant objects both in 3:2 and 4:3 MMR for three crucial instants of the migration:

(i) Beginning of simulation: The first point of the maximum on the curve of fractions of resonant objects versus time.

(ii) End of main decay phase: $t=10,50$ or $100 \mathrm{kyr}$ depending on the respective migration time-scale.

(iii) End of simulation: $t=250 \mathrm{kyr}$ for all of the cases.

The results show that, in general, the experiments with an eccentric Jupiter $\left(e_{\mathrm{J}}=0.06\right)$ result in lesser fractions of resonance

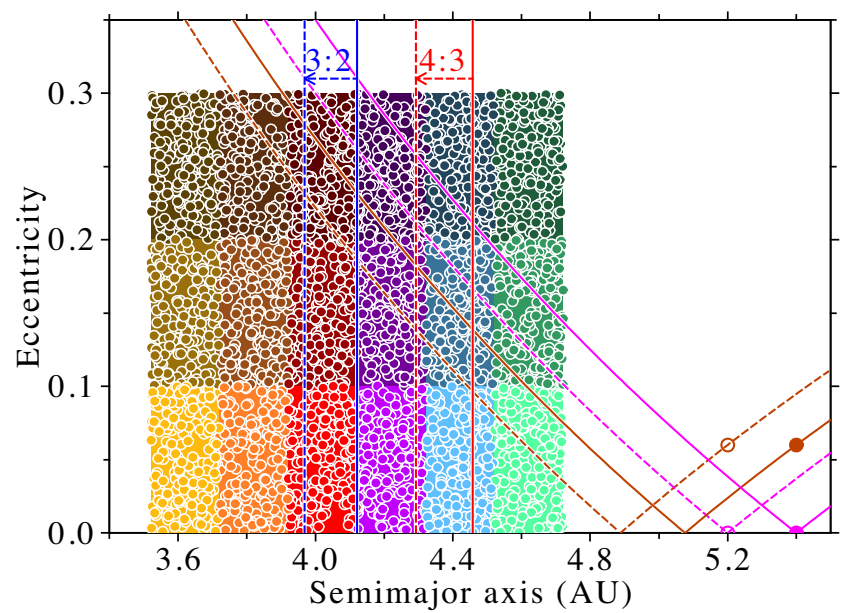

Figure 5. Initial distribution of the test particles in the $a \times e$ heliocentric space. The coloured rectangles represent $2 \mathrm{D}$ bins in this space. The circles stand for the distribution of test particles with their colours matching their original 2D bin. The initial and final states of Jupiter for both the circular and the eccentric cases are at 5.4 and $5.2 \mathrm{au}$. Vertical lines denote the loci of the resonances. Also shown are the aphelion and perihelion curves reaching the orbits of Jupiter. Globally, solid and dashed lines stand for the initial and final states, respectively, though the plot is a snapshot of the initial state.

capture. This is the same behaviour as that observed by comparing Figs 2 and 4. The only exception is the behaviour observed in the migration time-scale $100 \mathrm{kyr}$ for objects in 4:3 MMR. One can see this by comparing the solid error bars with the dotted ones for the instant for the main decay phase (central white column) and final migration (right-hand grey column).

Regarding new trappings in resonance during the main phase of the migration, this summary shows that most of the experiments present similar behaviour to that observed in Figs 2 and 4. However, some simulations present negligible new captures in resonance, like the example in Fig. 7.

\subsubsection{Jupiter leap experiments}

This section briefly addresses the results of an experiment developed to study the effects over the resonant populations due to an orbital leap of Jupiter. By orbital leap, we mean that Jupiter's orbit rises and shrinks within a short time relative to the overall time of migration. The reader is advised to keep in mind the distinction between an orbital leap and jump. Jumping Jupiter is the term employed for the overall rapid inward migration of Jupiter due to it having scattered an ice giant outwards.

The orbital leap study consists of a very simple experiment where 1000 test particles are distributed around the resonant locus. A numerical simulation is carried out with Jupiter fixed at 5.3 au during the first $2 \mathrm{kyr}$ so that the test particles in unstable orbits are removed. Analogously to the experiment described in Section 2, the test particles that exceed the region of interest (equation 2) are discarded. Then, after $2 \mathrm{kyr}$ the fraction of resonant objects is roughly constant. The initial semi-major axis of Jupiter, $5.3 \mathrm{au}$, is the median orbit between the initial and the final ones adopted for the migration experiments in this work. The eccentricity of Jupiter $e_{\mathrm{J}}$ is held equal to either 0.0 or 0.06 . Then, Jupiter is forced to migrate $\Delta a$ outward, within a time interval $(1 / 2) \Delta T$, and back inward within the ensuing time interval $(1 / 2) \Delta T$. 


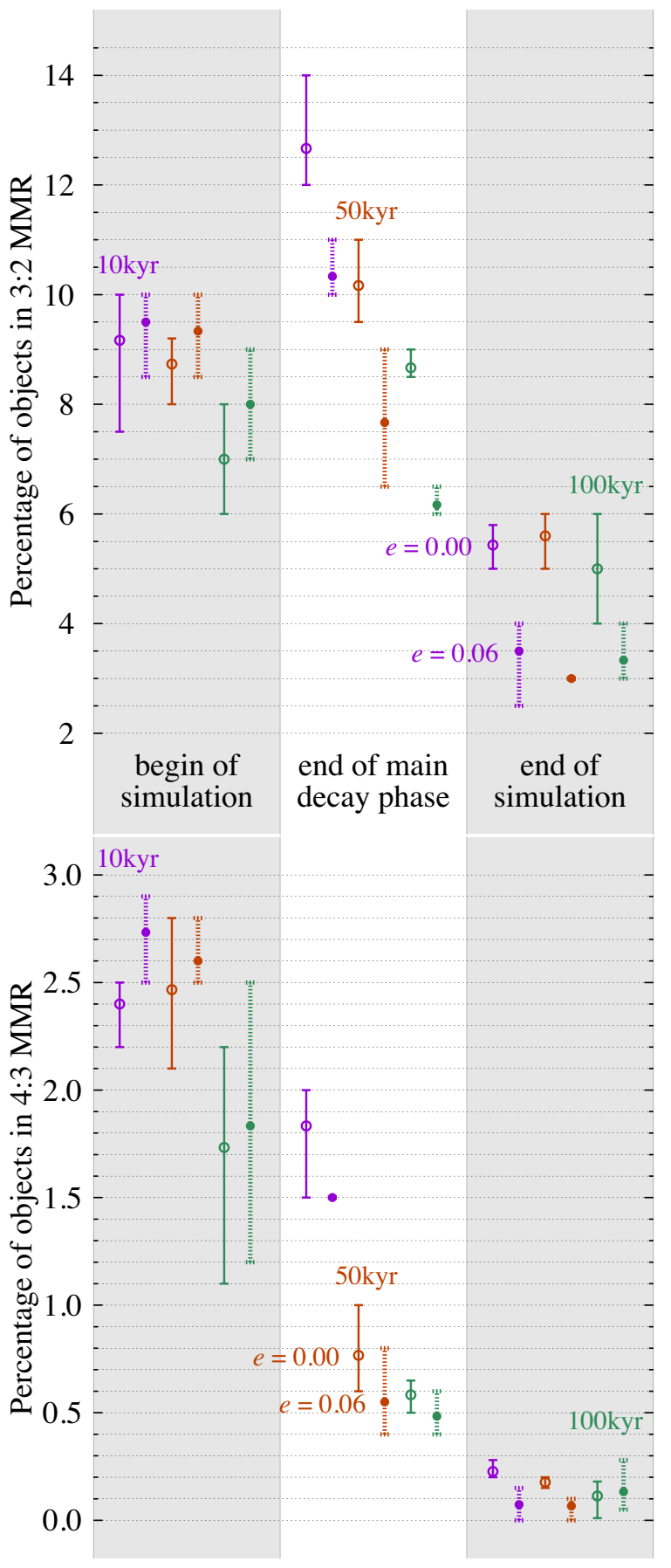

Figure 6. Summarized percentages of resonant objects at the three main stages of simulation. Top panel is for 3:2 MMR and the bottom one for 4:3. Vertical bar extremities stand for maxima and minima, evaluated for the three distinct simulations, and the circles stand for the mean value. The colours match the migration time-scale: $10 \mathrm{kyr}$ in magenta, $50 \mathrm{kyr}$ in brown and $100 \mathrm{kyr}$ in green. Solid error bars stand for the null Jovian eccentricity $\left(e_{\mathrm{J}}=0\right)$ whilst the dotted ones stand for experiments with $e_{\mathrm{J}}=0.6$.

Fig. 8 exemplifies such an experiment for $\Delta a=0.2 \mathrm{au}$, and $\Delta T=1.5 \mathrm{kyr}$ for the circular case, i.e. Jupiter's eccentricity is kept null during the whole simulation. First, the curves show that unstable test particles continue being discarded even after 2 kyr. Nevertheless, the rate of decrease at $t \approx 2 \mathrm{kyr}$ is very low and consequently negligible when compared to the resulting effects of the leap phenomenon. Indeed, as seen in Fig. 8, what is quite remarkable is

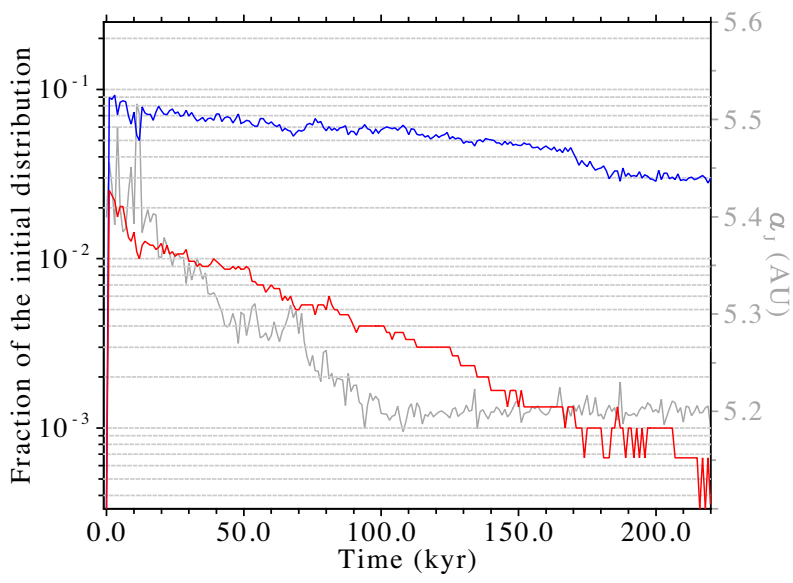

Figure 7. Fractions of asteroids in 3:2 in blue and 4:3 MMR in red for the simulation 100A6.

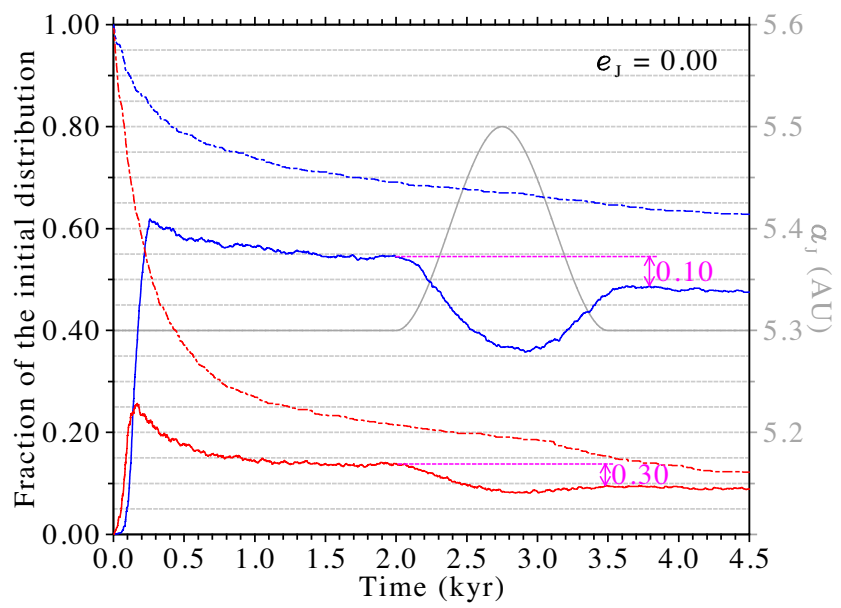

Figure 8. Results of an experiment with an orbital leap of Jupiter. The colours are the same as in Fig. 2. Additionally, dashed curves stand for the remaining fractions of test particles distributed around each resonance. The solid curves stand for the fractions of resonant objects. The label at the top right corner denotes the adopted eccentricity for Jupiter. Magenta denotes the net loss of resonant objects as a fraction of the resonant population at the initial instant of the leap.

the loss of resonant objects during the orbital leap due to the displacement of the resonant locus, and the ensuing recovery due to its receding, for both the resonances. At the end of the phenomenon, we observe a net loss of resonant objects both in 3:2 MMR (10 per cent) and 4:3 ( 30 per cent). As the locus of the resonance moves outward, some objects escape the resonance and a fraction of them surpass the region of interest and are consequently discarded. Hence, when the resonant locus recedes back to the original place, there are fewer objects to be captured back in resonance.

Of course, such a net loss varies according to the leap amplitude and duration. To check this, we carried out some experiments varying the four following parameters: $e_{\mathrm{J}}, \Delta a, \Delta T$ and the seed parameter adopted for a random distribution of the test particles. We performed a grid of 48 simulations for $e_{\mathrm{J}}=(0.00,0.06)$, $\Delta a=(0.1,0.2)$ au, $\Delta T=(0.25,0.5,1.0,1.5,2.0,3.0) \mathrm{kyr}$ and two distinct seeds. Fig. 9 summarizes the resulting data from all the 48 orbital leap experiments. Each symbol stands for the average net loss evaluated for two experiments. 


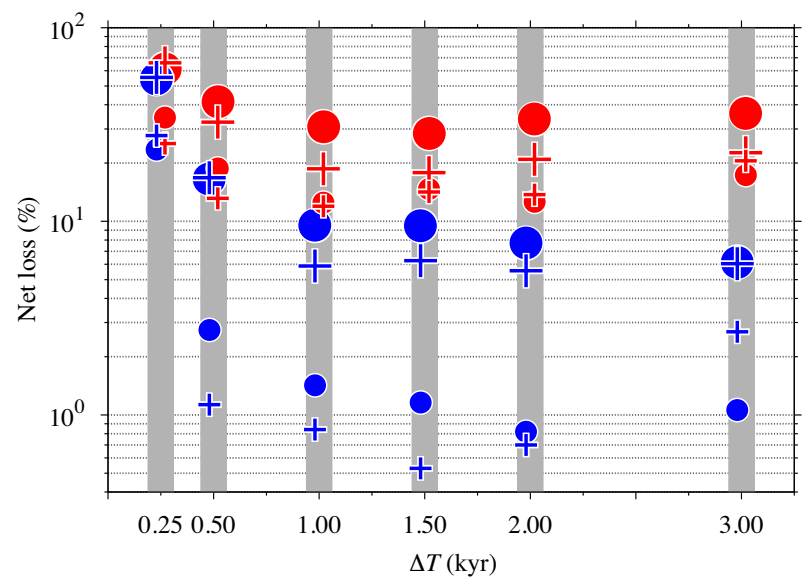

Figure 9. Summarized data for the whole set of orbital leap experiments. The blue and red colours match the resonances $3: 2$ and 4:3, respectively. Circles stand for circular cases, whilst crosses stand for cases with $e_{\mathrm{J}}=0.06$. Distinct symbol sizes match the leap amplitudes, small ones for $\Delta a=0.1$ au and the large ones for $\Delta a=0.2 \mathrm{au}$. To avoid hiding any symbols, we slightly displaced the blue ones to the left and the red ones to the right. The $\Delta T$ for each simulation is exactly that indicated by the grey bin that contains the symbol.

First, observe that the orbital leaps cause much more depletion to the population around the 4:3 MMR than to that of 3:2 MMR. This effect is somehow expected since the maximum eccentricity ${ }^{1}$ for objects in 4:3 MMR,

$e_{\max }=\frac{q_{\mathrm{J}}}{a_{4: 3}}-1$

is lower than the maximum eccentricity for objects in 3:2 MMR,

$e_{\max }=\frac{q_{\mathrm{J}}}{a_{3: 2}}-1$

Fig. 5 illustrates this.

Fig. 9 also shows the existence of an optimum value of $\Delta T$ for which the depletion is minimum. First, we explain what phenomenon causes more depletion for values of $\Delta T$ shorter than the optimum one. For shorter $\Delta T$, the displacement of the resonance locus is faster. Moreover, when the displacement of this locus is faster, the fraction of objects that escape the resonance is larger. Analogously for values of $\Delta T$ longer than the optimum one, if the time for the resonance locus to recede back is larger, then the fraction of non-resonant surviving objects is smaller. Hence, the optimum value of $\Delta T$ must be an equilibrium between these two phenomena.

These experiments reveal that the sequential leaps, even those of low amplitude, are responsible for the significant loss of resonant objects.

\subsection{Simulation final state}

For all of the experiments, we obtained reasonable fractions of objects trapped in 3:2 MMR. The plots of Fig. 10 show two examples of the final distribution: at the top for the circular case and at the bottom for the eccentric one. They are distributed within a bin of $\sim 0.2$ au width around the resonance locus. Also, they cover an eccentricity range from 0.0 up to 0.34 for Jupiter in a circular orbit.

\footnotetext{
${ }^{1}$ Maximum eccentricity is obtained by equating Jupiter's perihelion $q_{\mathrm{J}}$ to the aphelion of the asteroids $a(1+e)$.
}

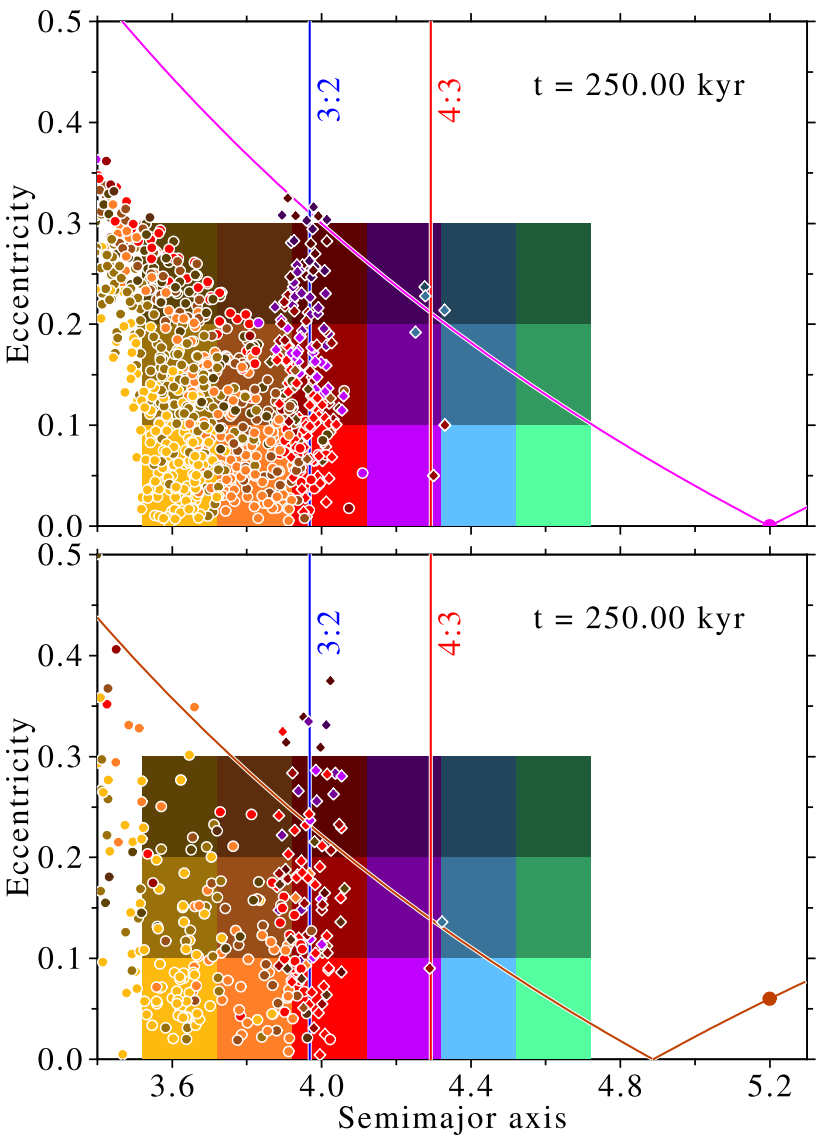

Figure 10. Analogous snapshots to that of Fig. 5, but for the final instant of the simulation. The top and bottom show the system's final state from the simulations 50A0 and 50A6, respectively. In these plots, the diamond symbols stand for the resonant objects.

For an eccentric Jupiter, a few objects had eccentricities excited to higher values than 0.34. On comparing the two plots of Fig. 10, notice the higher degree of eccentricity diffusion for the eccentric Jupiter case compared to the circular case. The colour intensity of the symbols allows us to infer that some of those objects trapped in resonance have had their eccentricity raised, and some have had their eccentricity reduced. By considering the colour of the symbols, one infers that few objects from the inner region of the primordial belt were trapped in resonance. The vast majority of the objects trapped in 3:2 MMR are from the red and violet bins. The outer regions were completely depleted.

\section{CONCLUSIONS}

This paper analyses the consequences for the outer region of the primordial main asteroid belt due to Jupiter's migration. The jumpingJupiter scenarios were considered using a synthetic migration algorithm, which mimics the time evolution of the semi-major axis shown in fig. 5 of Morbidelli et al. (2010). A more extended primordial asteroid belt is initially considered and the dynamical effects suffered during Jupiter's migration are studied. Jupiter's orbital eccentricity was kept constant by assuming either the value zero or 0.06 , for comparison.

The main conclusions can be discussed in terms of the following three aspects: the inner region to the locus of the 3:2 MMR, the $3: 2$ and the $4: 3$ resonant populations. The two resonant populations 
result from the capture in resonance of primordial objects, which is partially preserved during Jupiter's migration. Indeed, a substantial fraction of the objects captured in 3:2 MMR do migrate together with the respective resonance locus, even for Jupiter undergoing a truculent migration. Orbital leap experiments provide numerical estimates for the net loss of test particles for distinct featured leaps. Depending on the migration time-scale, the fraction of objects kept in 3:2 MMR varies from 2.5 up to $\gtrsim 5.5$ per cent. Even for objects in 4:3 MMR, in which the vast majority is lost throughout the migration phase, at least a fraction of $\sim 0.05$ per cent of the initial distribution remain captured.

It is important to clarify that this work evaluated the resonance trapping during the migration. Even after the end of the migration, long-term effects may maintain the leakage of resonant objects. Therefore, the trapping efficiencies are maximum values. One can also infer that the effects on the resonant populations do not substantially differ when comparing the results for Jupiter in an eccentric orbit for Jupiter in a circular orbit. On the other hand, by focusing on the distribution of the inner region to the locus of the 3:2 MMR, one notices that this region is much more depleted for the eccentric case. This result indicates two possibilities: (i) Jupiter may have undergone a substantially eccentric evolution or (ii) another mechanism must have depleted the current field between 3.6 au and $a_{3: 2}$ $\approx 3.97 \mathrm{au}$.

\section{ACKNOWLEDGEMENTS}

The authors gratefully acknowledge Fundação de Amparo à Pesquisa do Estado de São Paulo (FAPESP), Conselho Nacional de Desenvolvimento Científico e Tecnológico (CNPq) and Coordenação de Aperfeiçoamento de Pessoal de Nível Superior (CAPES). This work resulted from research funded by FAPESP, processes 2011/08171-3 and 2013/14418-8. This research has made use of data provided by the International Astronomical Union's Minor Planet Center. The authors also thank Prof ${ }^{\mathrm{a}}$. Vanessa Lima, Prof. Hazim Al-Qureshi and Julia Larroza for support with the grammar revision, and the referee Dr. Ramon Brasser, whose revision helped us to improve this paper significantly.

\section{REFERENCES}

Brasser R., Morbidelli A., Gomes R., Tsiganis K., Levison H. F., 2009, A\&A, 507, 1053

Everhart E., 1985, in Carusi A., Valsecchi G. B., eds, Proc. IAU Colloq 83, Dynamics of Comets: Their Origin and Evolution. Astrophysics and Space Science Library. Vol. 115, Reidel, Dordrecht, p.185
Fernandez J. A., Ip W.-H., 1984, Icarus, 58, 109

Franklin F. A., Lewis N. K., Soper P. R., Holman M. J., 2004, AJ, 128, 1391

Gomes R. S., Morbidelli A., Levison H. F., 2004, Icarus, 170, 492

Hahn J. M., Malhotra R., 1999, AJ, 117, 3041

Holman M., Williams G., Keys S., Rudenkog M., 2015, IAU Minor Planet Center. Available at: http://www.minorplanetcenter.net

IBM, 1954, Specifications for the IBM Mathematical FORmula TRANSlating system. Preliminary report, IBM Corp., Programming Research Group, Applied Sciences Division, New York, NY

Minton D. A., Malhotra R., 2009, Nature, 457, 1109

Morbidelli A., Levison H. F., Tsiganis K., Gomes R., 2005, Nature, 435, 462

Morbidelli A., Brasser R., Tsiganis K., Gomes R., Levison H. F., 2009 A\&A, 507, 1041

Morbidelli A., Brasser R., Gomes R., Levison H. F., Tsiganis K., 2010, AJ, 140,1391

Murray C. D., Dermott S. F., 1999, Solar System Dynamics, 1st edn. Cambridge Univ. Press, Cambridge

Nesvorný D., 2011, ApJ, 742, L22

Ritchie D., Kernighan B., 1978, The C Programming Language, 1st edn. Prentice-Hall International, Inc, London, UK

Roig F., Nesvorný D., 2015, AJ, 150, 186

Tsiganis K., Gomes R., Morbidelli A., Levison H. F., 2005, Nature, 435, 459

Walsh K. J., Morbidelli A., Raymond S. N., O’Brien D. P., Mandell A. M. 2011, Nature, 475, 206

Ward W. R., 1997, Icarus, 126, 261

\section{SUPPORTING INFORMATION}

Supplementary data are available at MNRAS online.

Appendix A. Jumping-Jupiter mimicking algorithm

Please note: Oxford University Press is not responsible for the content or functionality of any supporting materials supplied by the authors. Any queries (other than missing material) should be directed to the corresponding author for the article.

This paper has been typeset from a $\mathrm{T}_{\mathrm{E}} \mathrm{X} / \mathrm{L} \mathrm{T} \mathrm{E} X$ file prepared by the author. 